\title{
Searching for isolated black holes in the Milky Way
}

\section{Francesca Scarcella, ${ }^{a, *}$ Daniele Gaggero ${ }^{a}$ and Juan García-Bellido ${ }^{a}$}

${ }^{a}$ Instituto de Física Teórica UAM-CSIC, Campus de Cantoblanco, E-28049 Madrid, Spain

E-mail: francesca.scarcella@uam.es

If Primordial Black Holes constitute a significant component of the Dark Matter, we expect a fraction of the population to be present at the center of our Galaxy. This region is rich in dense molecular clouds. Black holes located within a cloud will accrete the dense interstellar gas, which will emit radiation during the process, providing a possible channel for detection. Here we discuss the scenario in which the Primordial Black Holes masses follow a multimodal mass function, as motivated by the thermal history of the universe, and compare it to the monochromatic case.

$37^{\text {th }}$ International Cosmic Ray Conference (ICRC 2021)

July 12 th - 23rd, 2021

Online - Berlin, Germany

\footnotetext{
${ }^{*}$ Presenter
} 


\section{Introduction}

Primordial Black Holes (PBHs) have been proposed as a Dark Matter (DM) candidate. These compact objects can be formed in the early Universe in a very wide range of masses. In particular, the possibility of PBHs existing in the solar-mass range has attracted much attention following the recent observations of binary black hole mergers.

A complementary channel to observe PBHs in this mass range could be provided by the accretion of dense gas from molecular clouds. The accreted gas is expected to emit non-thermal radiation (mainly from bremsstrahlung and inverse Compton scattering), which could be detected in the X-ray and radio bands. This process could allow us to detect the presence of isolated PBH within our own Galaxy. So far, the only observations of isolated BHs in the Milky Way have been obtained through microlensing (see e.g. [1], [2] and [3] for a discussion of the constraints that can be derived from these observations).

On the other hand, no isolated $\mathrm{BH}$ has yet been identified through the process of accretion. Surveys in the X-ray and Radio band have been used to place bounds the fraction of PBHs in DM for PBH with masses of $O\left(10 \mathrm{M}_{\odot}\right)$, using log-normal and power-law mass distributions [4]. We consider here instead more complex multi-modal mass function [5], motivated by the thermal history of the early Universe: corresponding to drops in the pressure of the relativistic fluid that occur at particular cosmological epochs, the density contrast threshold for PBH formation is lowered. These drops, the most significant of which corresponds to the binding of quarks into hadrons, induce exponential boosts in the PBH formation rate. Taking this into account and starting from a nearto-flat power spectrum for the density fluctuations, one obtains a PBH mass distribution such as the one shown in Fig.1. It features a peak between 10 and $100 \mathrm{M}_{\odot}$, in the range of the Ligo-Virgo observations, but its most prominent peak is around $1 \mathrm{M}_{\odot}$.

Here we discuss the possibility of detecting PBH through the accretion process in the Central Molecular Zone (CMZ). This region of the Milky Way is characterized by the presence of large and dense molecular clouds. It is thus a natural target for this type of search. The accretion process is described using a state-of-the art model [6]. This discussion is a preliminary to a forthcoming work by the authors.

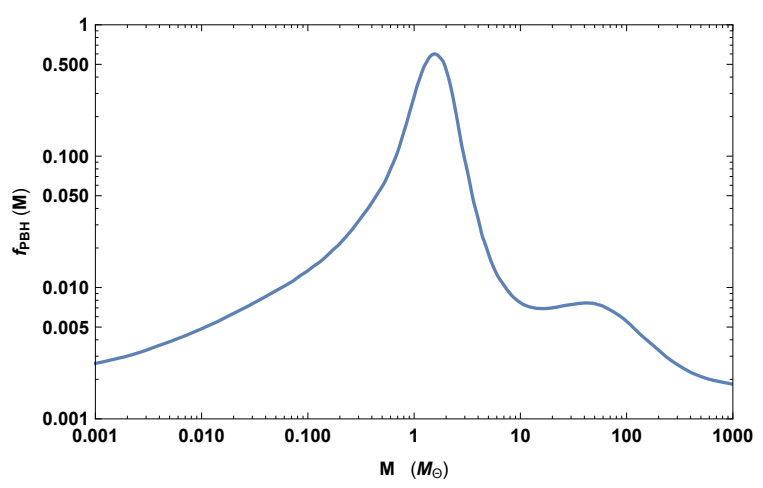

Figure 1: The mass spectrum of PBHs considered in this work [5]. 


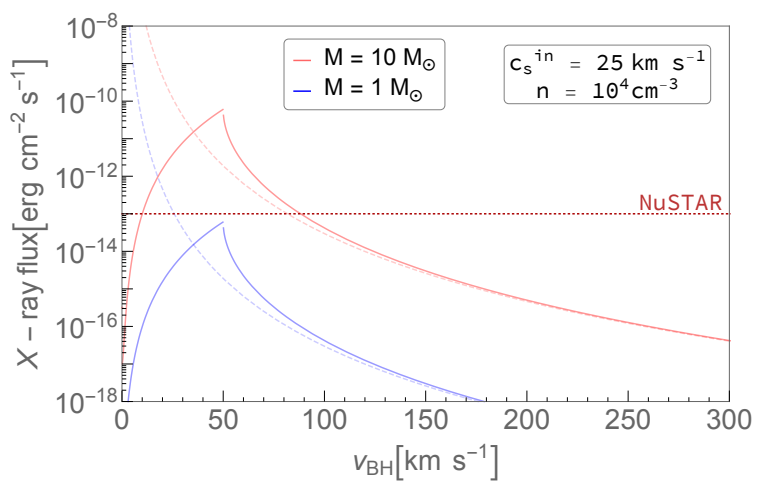

Figure 2: We show the X-ray flux predicted using the PR model (solid), as a function of the BH speed relative to the cloud. We also show the BHL (dashed) prediction. The two models agree at high velocity, but predictions differ by many orders of magnitude in the low velocity range.

\section{Theoretical setup}

\subsection{The accretion process and emission of radiation}

For our purpose, it is important to accurately model of the accretion process. We rely on the state-of-the-art model due to Park and Ricotti (PR) [6-8], based on hydro-dynamical simulations including radiative feedback. Its main correction to the standard Bondi-Hoyle-Littleton (BHL) $[9,10]$ picture is that the accretion rate turns out to be strongly suppressed when the $\mathrm{BH}$ travels through the interstellar medium at low speeds. This suppression is due to the formation of a shock front ahead of the ionized region surrounding the $\mathrm{BH}$ (the gas is ionized by the radiation emitted durig the accretion process). This phenomenon occurs below a threshold velocity that is determined by the temperature of the ionized gas: $\mathrm{v}_{\text {peak }}=2 c_{\mathrm{s}}^{\text {in }}$, where $c_{\mathrm{s}}^{\text {in }}$ is the sound speed of the ionized gas (which we expect to be of the order of a few tens of $\mathrm{km} / \mathrm{s}$ ). Thus, the accretion rate displays a peak value, in contrast to the BHL rate which increases monotonically as the velocity lowers. We model the radiation emission assuming a Radiatively Inefficient Accretion Flow (RIAF) (see Ref. [8] for details).

In Fig. 2 we show the X-ray flux at Earth for two BHs of different mass accreting from a typical molecular cloud at the Galactic centre (GC), as a function of their velocity relative to the cloud. Comparing the predicted flux to the threshold of the NuSTAR survey of the GC [11], we can notice that $\mathrm{BH}$ of $\sim 1 \mathrm{M}_{\odot}$ or less are not detectable for any value of the relative speed. Notice also how the BHL model (dashed lines ${ }^{1}$ ) fails to capture this threshold effect. This property is particularly relevant in the context of the mass function we are considering. The threshold mass can decrease by a factor of a few if the cloud is denser or if the ionized sound speed $c_{\mathrm{s}}^{\text {in }}$ is smaller. For instance, we find a threshold mass of approximately $.2 \mathrm{M}_{\odot}$ for $c_{\mathrm{s}}^{\mathrm{in}}=10 \mathrm{~km} / \mathrm{s}$ (in this case, the location of the peak is shifted to the left, so only very slow PBHs are visible).

\footnotetext{
${ }^{1}$ We do not introduce the usual suppression factor of the BHL rate, to allow for a more direct comparison of the models
} 


\subsection{Molecular clouds}

Our target region, the CMZ, is a cloud complex with an approximately cylindrical shape that extends up to $\sim 200 \mathrm{pc}$ away from the GC. A component of the molecular clouds in this region present very high number densities, $n \sim 10^{3} .5-10^{4} \mathrm{~cm}^{-3}$ [12]. In order to capture the presence of more diffuse, less dense clouds and of denser, more concentrated clumps, we model the density distribution of the clouds with a power law, $P(n) \propto n^{-\beta}$. Allowing for very dense clumps means that the threshold effect discussed in the previous section becomes blurred: light PBHs can become visible if the gas is dense enough. Finally, we assume the clouds to be distributed uniformly in the region.

\subsection{PBH spacial distribution}

An important quantity to be estimated is the number of PBHs that are expected to be found within the CMZ. Unfortunately, the distribution of the DM is poorly constrained near the GC. Here we assume a Navarro-Frenk-White (NFW) profile and integrate it over the volume of the CMZ to obtain the total number of PBHs. We then assume the BHs to be distributed uniformly within the region and that their positions are not correlated with those of the clouds.

\subsection{PBH speed distribution}

We model the velocities of the PBH population with a Maxwell-Boltzmann distribution. The mean value of the distribution is an important parameter, given the strong dependence from it of the accretion rate. Using Eddington's inversion method results in values of about $50 \mathrm{~km} / \mathrm{s}$, corresponding to near-peak accretion rates. On the other hand, observations of stars near the GC show a higher velocity dispersion, around $130 \mathrm{~km} / \mathrm{s}$ [13]. Here we treat the mean velocity as a free parameter.

\section{Results}

In this section we present our preliminary results as bounds on the fraction $f_{\mathrm{PBH}}$ of $\mathrm{PBHs}$ in DM. In particular, we consider emission in the X-ray band and obtain the bound by comparison with the catalog of the NuSTAR survey [11]. NuSTAR has observed about 70 sources in the GC, most of which are compatible with being accreting compact objects. We present the bounds in Fig. 3. The two panels correspond to different modelling of the clouds and gas. In particular, we use different values for:

- the power-law index $\beta$ of the density distribution (on the left, slightly more dense clumps are present)

- the sound speed $c_{\mathrm{s}}^{\text {in }}$ of the ionised gas around the black hole, 



Figure 3: Bounds on $f_{\mathrm{PBH}}$ obtained by comparison with the catalog of the NuSTAR survey [11]. The two panels correspond to an optimistic and a conservative case with respect to the description of the accreted gas. Diffferent colors correspond to different values of the average PBH speed. In solid lines, the results for the multimodal mass distribution scenario. In dashed lanes, the monochromatic case.

the latter being the most relevant of the two parameters. In different colors we show different values for the average PBH speed $\mu_{\mathrm{BH}}$. As expected, we predict more sources and obtain a stronger bound when this is close to the value corresponding to peak accretion rate: $\mathrm{v}_{\text {peak }}=2 c_{\mathrm{s}}^{\mathrm{in}}$.

The dashed lines depict the bound obtained in the monochromatic case, as a function of the PBH mass. Depending on the choice of parameters, we can obtain strong bounds on $f_{\mathrm{PBH}}$ for masses above $\sim 1 \mathrm{M}_{\odot}$ or above $\sim 10 \mathrm{M}_{\odot}$ sun. The solid lines refer to the multi-modal mass distribution which is the object of our study. We can notice how the result is dominated by the peak of the mass distribution, at $\mathrm{M} \sim 1 \mathrm{M}_{\odot}$. The results show an important variability depending on the choice of parameters. In some regions of the parameter space, and in particular when velocities are assumed to be low, we predict a large number of sources. For other combinations of the parameters, the number of sources is lower than the number of objects in the NuSTAR catalog and no bound can be placed.

\section{Caveats}

Aspects of the problem to be further analyzed include:

- The number of PBH in the CMZ region. We have fixed this number assuming an NFW profile, but large uncertainties are associated to the DM distribution in the inner region of the Galaxy.

- We have assumed, for simplicity, that the PBHs are distributed uniformly. PBHs are, however, expected to gather in clusters. If such clusters are present in the CMZ, this could alter the results significantly. 


\section{Conclusions}

We have discussed the possibility of detecting PBHs through the process of accretion from molecular clouds in central region of the Galaxy. In particular, we have considered a PBH population described by a multi-modal mass function as motivated by the thermal history of the early Universe. We have studied in particular the X-ray emission and compared the predicted fluxes with the NuSTAR survey. We have found that the PBHs of $\sim 1 \mathrm{M}_{\odot}$ are only visible if accreting from very dense clouds and for a small range of velocities. Since the mass distribution that we are taking into account in the present work is actually dominated by these small PBHs, it is very difficult to place a solid constraint on the fraction of PBH in DM within this scenario. On the other hand, accreting BHs belonging to the subdominant, more massive component (tens of solar masses), are expected to emit significant radiation for a wide range of velocities. An interesting window exists, therefore, for the detection of these objects. In particular, our result calls for an accurate analysis of existing data to determine whether such objects have already been detected.

\section{References}

[1] Lukasz Wyrzykowski and Ilya Mandel. Constraining the masses of microlensing black holes and the mass gap with Gaia DR2. Astron. Astrophys., 636:A20, 2020.

[2] Numa Karolinski and Wei Zhu. Detecting isolated stellar-mass black holes in the absence of microlensing parallax effect. Mon. Not. Roy. Astron. Soc., 498(1):L25-L30, 2020.

[3] Josh Calcino, Juan García-Bellido, and Tamara M Davis. Updating the MACHO fraction of the Milky Way dark halowith improved mass models. Monthly Notices of the Royal Astronomical Society, 479(3):2889-2905, 052018.

[4] Julien Manshanden, Daniele Gaggero, Gianfranco Bertone, Riley M. T. Connors, and Massimo Ricotti. Multi-wavelength astronomical searches for primordial black holes. JCAP, 06:026, 2019.

[5] Bernard Carr, Sebastien Clesse, Juan García-Bellido, and Florian Kühnel. Cosmic conundra explained by thermal history and primordial black holes. Phys. Dark Univ., 31:100755, 2021.

[6] KwangHo Park and Massimo Ricotti. Accretion onto Black Holes from Large Scales Regulated by Radiative Feedback. III. Enhanced Luminosity of Intermediate Mass Black Holes Moving at Supersonic Speeds. Astrophys. J., 767:163, 2013.

[7] Kazuyuki Sugimura and Massimo Ricotti. Structure and Instability of the Ionization Fronts around Moving Black Holes. Mon. Not. Roy. Astron. Soc., 495(3):2966-2978, 2020.

[8] Francesca Scarcella, Daniele Gaggero, Riley Connors, Julien Manshanden, Massimo Ricotti, and Gianfranco Bertone. Multi-wavelength detectability of isolated black holes in the Milky Way. 122020. 
[9] F. Hoyle and R. A. Lyttleton. The effect of interstellar matter on climatic variation. Proceedings of the Cambridge Philosophical Society, 35:405, 1939.

[10] H. Bondi and F. Hoyle. On the mechanism of accretion by stars. MNRAS, 104:273, 1944.

[11] JaeSub Hong et al. NuSTAR Hard X-ray Survey of the Galactic Center Region II: X-ray Point Sources. Astrophys. J., 825(2):132, 2016.

[12] Katia Ferriere, William Gillard, and Pierre Jean. Spatial distribution of interstellar gas in the innermost $3 \mathrm{kpc}$ of our Galaxy. Astron. Astrophys., 467:611-627, 2007.

[13] Jason L Sanders, Leigh Smith, N Wyn Evans, and Philip Lucas. Transverse kinematics of the galactic bar-bulge from vvv and gaia. Monthly Notices of the Royal Astronomical Society, 487(4):5188-5208, Jun 2019. 